\title{
Learning by Doing: Evaluation of an Educational VR Application for the Care of Schizophrenic Patients
}

Soojeong Yoo

School of Architecture, Design

and Planning

University of Sydney

Sydney, Australia

soojeong.yoo@sydney.edu.au
Youngho Lee

Dept. of Computer Engineering

Mokpo National University

Jeonnam, South Korea

youngho@ce.mokpo.ac.kr

Sunkyung Kim

Dept. of Nursing

Mokpo National University

Jeonnam, South Korea

skkim@mokpo.ac.kr

Permission to make digital or hard copies of part or all of this work for personal or

classroom use is granted without fee provided that copies are not made or distributed for profit or commercial advantage and that copies bear this notice and the full citation on the first page. Copyrights for third-party components of this work must be honored. For all other uses, contact the owner/author(s).

CHI '20 Extended Abstracts, April 25-30, 2020, Honolulu, HI, USA.

(c) 2020 Copyright is held by the author/owner(s).

ACM ISBN 978-1-4503-6819-3/20/04

DOI: https://doi.org/10.1145/3334480.3382851

\begin{abstract}
Nursing students learn a variety of skills to work in a clinic, such as dealing with patients with particular requirements, handling expensive equipment, and assisting doctors with treatments. However, a specific situation may require handson experience that cannot be easily conveyed through a textbook or a video, such as caring for a schizophrenic patient. Simulation has been considered as an effective method to replace observation-based clinical placement to overcome safety issues. In this paper, we investigate the possibility of employing a virtual reality (VR) learning platform for nursing students to learn how to care for schizophrenic patients. Using 360-degree video and a head-mounted display (HMD), students experienced virtual patients who have schizophrenia portrayed by professional actors. Our key contribution is in the insights about the design of educational VR applications, highlighting the potential value of VR for training students with non-technical backgrounds.
\end{abstract}

\section{Author Keywords}

Virtual reality; Education; Nursing; $360^{\circ}$ Video; HMD; Schizophrenic

Introduction

Schizophrenia is a mental illnesses which affects people worldwide [2]. The symptoms of the disorder include a 


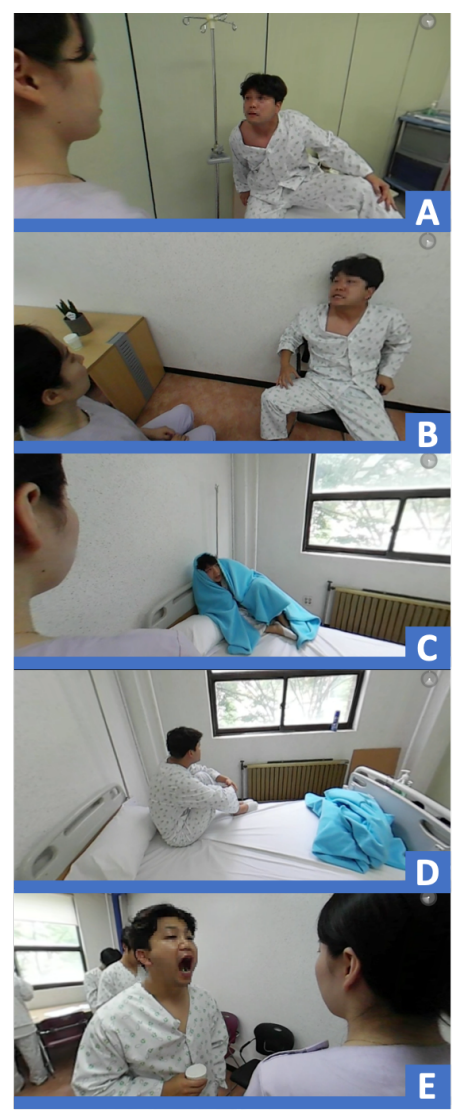

Figure 1: Actor pretending to be a patient. Five different scenarios on five schizophrenia symptoms. From the top: (A) risk of violation; $(B)$ auditory hallucination; $(C)$ visual hallucination; (D) delusion; and (E) risk of suicide. range of cognitive, behavioural, and emotional problems that usually involve delusions, hallucinations, and aggression. Given the complexity of illness, nurses in psychiatric units require sufficient training and case experience; as a lack of training experience may compromise the quality of care and result in negative outcomes for both the patients and the nurses [1]. Most clinical placements for mental health nursing are currently limited to observation to ensure the safety of both the patients and the students [7].

An alternative to clinical placement is a simulation, which allows participants to role-play realistic clinical situations without the risks present in the real world $[6,11,4]$. The simulation involves actors who portray the symptoms of the patients with mental illness. However, these simulations can require a lot of setting up and planning, while not being accessible to a broad range of nursing students.

Building on the success of simulations, virtual reality (VR) can allow users to be fully immersed in the virtual world. Recently, with the growing maturity of VR technology and falling hardware costs, VR has begun to emerge as a viable technology for educational simulations. For instance, students could learn how to use expensive equipment without the risks associated with real life $[9,5]$.

Despite the effectiveness of VR as a educational tool, most nursing schools rely on videos from the web [10] or use desktop simulators during class, while the implementation of practical training using immersive VR has been rare [8, 3].

In this paper, we present the design and implementation of an education platform based on an immersive VR system, tailored for nursing students. Through a head-mounted display (HMD), we showed students five different scenarios and situations, filmed with a $360^{\circ}$ camera. Each scenario was developed based on clinical conditions in which registered nurses witnessed the problematic behavior of patients who were admitted to the psychiatric ward in an acute setting. After reflecting on the symptoms of schizophrenia and hospital environments in acute settings, the scripts were written by a researcher and reviewed by a team of experts (two professors and one mental health nursing professional) and then edited for realism and length of the videos. To evaluate the implementation of the VR system and to gain insights into the design of such systems for nursing education, we conducted a study where 60 participants experienced five scenarios on schizophrenic patients.

\section{Study Design}

Scenarios

The scenarios of mental illness patients in an acute setting were developed based on clinical situations with the most possible problematic behaviors. The video scripts were written by a professor in a department of nursing and reviewed by a team of experts (two professors and one mental health nursing professional) in our university and then properly edited to obtain a suitable level of realism and an appropriate length. Professional actors were hired to play different roles, and each scene was recorded using a $360^{\circ}$ camera (Samsung Gear $360^{1}$ ). The scenes were first rehearsed, and then officially filmed after implementing the feedback from experts, who suggested to improve facial expressions and the interaction between actors.

The final video clips were validated in terms of closeness to reality by two mental health nursing professionals and one nurse practitioner working in a psychiatric unit. The research team developed questions as tasks at several decision points to test participants' knowledge and capability

${ }^{1}$ https://www.samsung.com/global/galaxy/gear-360/ 


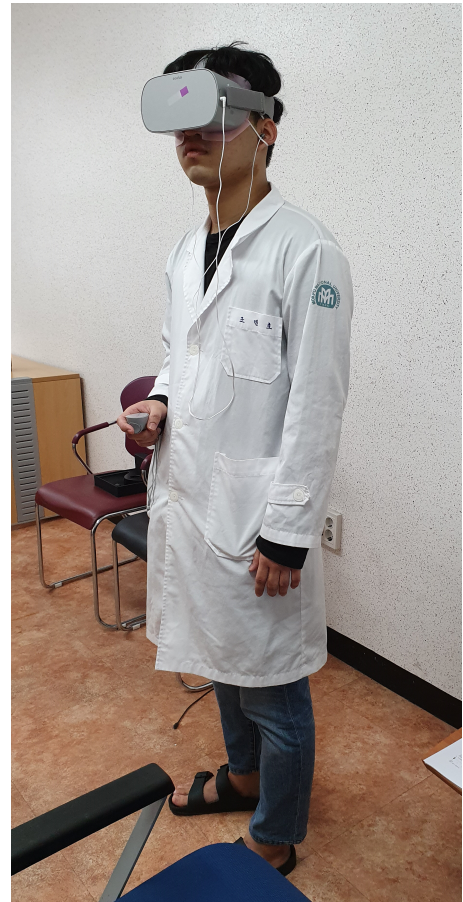

Figure 2: A nursing student wears a HMD and holds a controller in his right hand. for optimal decision-making and critical thinking. The questionnaires were also evaluated and reviewed by a group of experts in terms of clinical relatedness and level of difficulty for testing students.

Based on this, five different scenarios were developed that incorporated five various symptoms of schizophrenia (1) risk of violation, (2) auditory hallucination, (3) visual hallucination, (4) delusion, and (5) medication of refusal (Figure 1). Each scenario ranged from 60 to 90 seconds in length.

The risk of violence is a situation in which a patient is angry and tends to be violent toward a nurse or another patient. An auditory hallucination is a circumstance in which a patient becomes violent just by imagining a hostile relationship with another person. During a visual hallucination, a patient sees people, objects, or buildings that do not exist. During delusion, an idiosyncratic belief or impression that is firmly maintained despite being contradicted by what is generally accepted as reality or rational argument, typically a symptom of mental disorder. While in medication of refusal, the patient strongly refuses to take medication due to the delusion that medicine would harm her/him, making their condition worse.

\section{Implementation}

We used the Oculus $\mathrm{Go}^{2}$ VR headset for the usability evaluation. The device was equipped with a $2560 \times 1440$ resolution LCD and a hand controller as an input device. Gear 360 uses two fish-eye lenses to acquire videos with a resolution of $4096 \times 2160(24 \mathrm{f} / \mathrm{s})$ and uses the H.265 video compression standard. To develop our software, we used the Unity game engine, a commonly used engine for games and VR program development.

\footnotetext{
${ }^{2}$ https://www.oculus.com/go/
}

When students wore the HMD (Figure 2), they were able to control several features with the handheld controller. Figure 3 shows a snapshot of a video displayed on Oculus Go. The students were also able to pause, play, and stop the videos through the controller.

\section{Study Process}

A usability test was conducted to evaluate the ease of use and usefulness of VR programs for simulation training of nursing students using the scenario of patients with mental illness, schizophrenia. Before the VR simulation, detailed guidance about how to use the HMD and controller was presented in three phases that took about 15 to 20 minutes in total. In the beginning, a step-by-step introduction about where to look and which buttons to click was given by a research assistant. Following this, the researcher demonstrated how to handle the controller while explaining the sequences of the VR simulation using visualised pictures and text.

At the very end of all five scenarios, we collected qualitative feedback through interviews, to seek participants' perception of the using VR simulation application to use as a potential value of VR for training schizophrenic patients.

In this time, the researcher asked three questions to each participant:

- What is your overall feeling about this virtual simulation training system in regards to the care of schizophrenic patients?

- How do you find this way of learning? Would it be helpful for your future clinical practice?

- Was the VR system easy to use? 


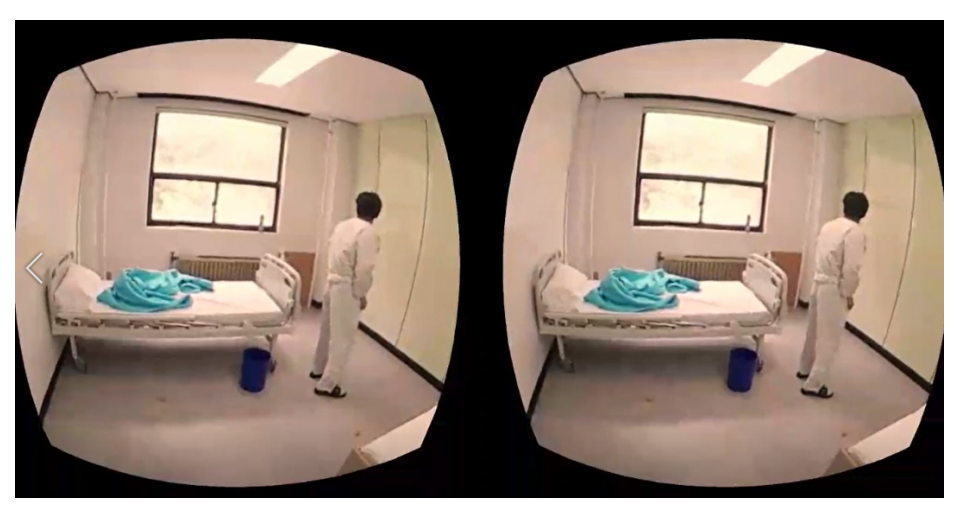

Figure 3: A video played on the Oculus Go

\section{Results}

Participants

For this study, we recruited 60 nursing students (46 female and 14 male, mean age 23.6) from Mokpo National University in South Korea. All procedures in this study were approved by the university's Institutional Review Board (IRB No. MNUIRB-20190722-SB-005-01). The participants were all seniors who completed the majority of their clinical placement training. Four students out of $60(6.7 \%)$ experienced VR before.

As shown in Figure 4, regarding the general perception of the VR simulation for the treatment of patients with schizophrenia, half of our participants reported that it felt realistic $(\mathrm{n}=30)$. Several participants' commented "It was like I was actually in the room so I felt fearful when the actors behaved violent and came close to me". Participants also mentioned that it was an interesting experience and useful to experience a scenario of patients with more complicated mental health issues. Additionally, 6 participants mentioned that it would be effective for learning.

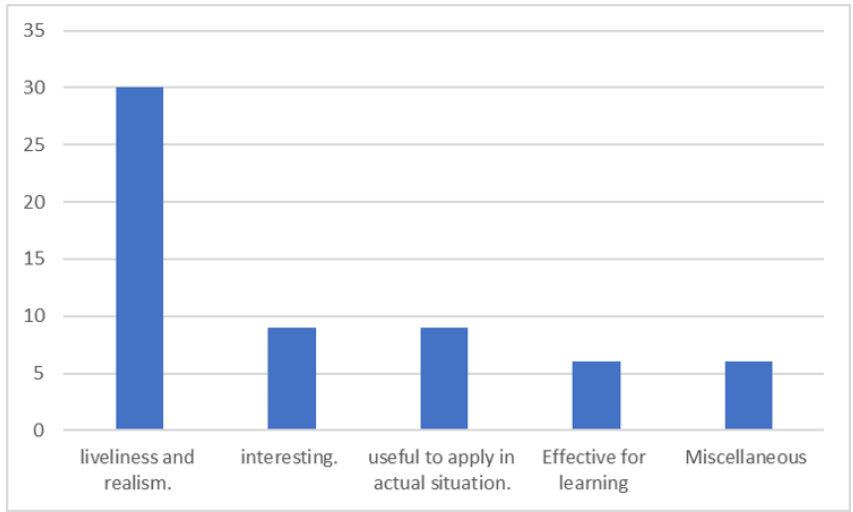

Figure 4: The responses to the opinion poll on the students overall feeling of the test.

All participants agreed that this VR simulation program would eventually benefit their future clinical practice. Specifically, participants attributed the educational effectiveness of the program to its high level of immersion. Several participants commented on the educational benefits of the VR simulation ("This program provides a better understanding of the symptom-related nursing process for patients with schizophrenia."). Other participants made comments such as: "I may never have the courage to face patients with such mental conditions during clinical placement". Some participants appreciated the safe learning environment of this VR simulation, stating exposure to dangerous clinical situations without risk is a great benefit of this learning program ("I felt safe enough to attempt communication with patients in this VR simulation").

For the last questions on VR usability to the students, as shown in Figure 5, forty students agreed that the operation was straightforward. However, several students complained about difficulties in manipulating the video and responding 
to the questions using the controller. We predict that these problems will be solved when a VR user interface is developed that is easier to handle and made available to anyone.

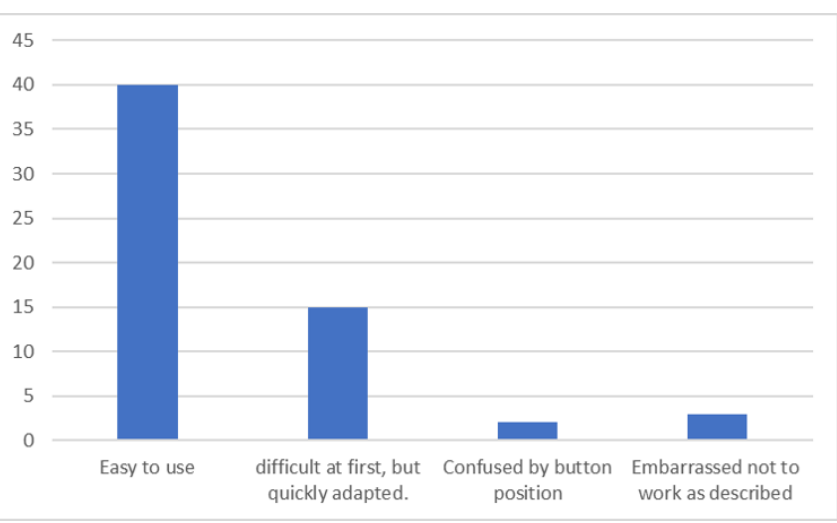

Figure 5: The responses to the opinion poll on the usability of our system.

\section{Implication}

Findings of this study identified that the virtual environment built using 360 degree and HMD could offer high realism in simulation education.

The symptom of patients with mental illness would be portrayed better using professional actors. As previous work mentioned, there have been quality control issues using actors in training simulations [4]. Additionally, the simulation environment would be safer using video that filmed in a controlled environment with well developed scenarios which are reviewed by multiple experts.

It is important to improve students' confidence in facing patients with problematic behavior. Through experiencing clinical situations in a simulation, better attitude towards mentally ill patients can be achieved.
Unlike traditional 2D video, 3D virtual simulation using 360 degree video could replace traditional simulation using actors thanks to its ability to create a life-like world. Since the realism is one important matter in simulation training, VR simulation could be an excellent method in nursing simulation providing students experience with high realism.

\section{Limitation}

We encountered several limitations from our initial study.

Some students experienced motion sickness suggesting a warning not to use the prototype longer than 10 to 15 minutes at a time. The weight of the HMD and its resolution may have also been contributing factors to the motion sickness.

Students also mentioned that wearing a HMD made it difficult to communicate and share their experience with other students and tutors. In general, VR systems are developed to allow only one person to visualise a scene, without the possibility to interact with other users. To be used for educational purposes, further work could focus on creating a multi-user environment for classrooms along with LMS integration which can easily and effectively allow teachers to interact and check their students' progress.

\section{Conclusion and Future works}

In this paper, we investigated the requirements of students education, and developed an educational VR system that allowed us to show the student nurses $360^{\circ}$ videos of reallife scenarios on schizophrenic patients through a VR HMD. This study suggested that an important future direction is exploring different user interfaces for getting help with these issues, and a learning management system, which can help increase the usability and effectiveness of our educational platform for nursing students. The voice and gesture interface will allow a student to respond using voice or gesture 
responses to the situation.

\section{Acknowledgements}

This work was supported by the National Research Foundation of Korea (NRF) grant funded by the Korea government (MSIT) (No. NRF-2019R1G1A1006737) and (No. NRF-2018R1D1A1B07048247)

\section{REFERENCES}

[1] American Psychiatric Association and others. 2013. Diagnostic and statistical manual of mental disorders. BMC Med 17 (2013), 133-137.

[2] Fiona J Charlson, Alize J Ferrari, Damian F Santomauro, Sandra Diminic, Emily Stockings, James G Scott, John J McGrath, and Harvey A Whiteford. 2018. Global epidemiology and burden of schizophrenia: findings from the global burden of disease study 2016. Schizophrenia bulletin 44, 6 (2018), 1195-1203.

[3] Cynthia L. Foronda, Krysia Warren Hudson, and Chakra Budhathoki. 2017. Use of Virtual Simulation to Impact Nursing Students' Cognitive and Affective Knowledge of Evidence-Based Practice. Worldviews on Evidence-Based Nursing 14, 2 (2017), 168-170. DOI : http://dx.doi .org/10.1111/wvn.12207

[4] Sun Kyung Kim, Mi Ran Eom, and Mi-hyeon Park. 2019. Effects of Nursing Education Using Virtual Reality: A Systematic Review. Journal of the Korea Contents Association 19, 2 (2019), 661-670. DOI : http://dx.doi.org/10.5392/JKCA.2019.19.02.661

[5] Mehmet Kizil. 2003. Virtual reality applications in the Australian minerals industry. Application of Computers and Operations Research in the Minerals Industries October (2003), 569-574.
[6] Patrick Lavoie and Sean P Clarke. 2017. Simulation in nursing education. Nursing management 48, 2 (2017), 16-17.

[7] Kyung Choon Lim. 2015. Planning and applying simulation-based practice for the achievement of program outcomes in nursing students. The Journal of Korean Academic Society of Nursing Education 21, 3 (2015), 393-405.

[8] Pamela C. Smith and Bernita K. Hamilton. 2015. The effects of virtual reality simulation as a teaching strategy for skills preparation in nursing students. Clinical Simulation in Nursing 11, 1 (2015), 52-58. DOI :

http://dx.doi.org/10.1016/j.ecns.2014.10.001

[9] K. Tanoue, S. leiri, K. Konishi, T. Yasunaga, K. Okazaki, S. Yamaguchi, D. Yoshida, Y. Kakeji, and M. Hashizume. 2008. Effectiveness of endoscopic surgery training for medical students using a virtual reality simulator versus a box trainer: A randomized controlled trial. Surgical Endoscopy and Other Interventional Techniques 22, 4 (2008), 985-990. DOI :

http://dx.doi.org/10.1007/s00464-007-9554-8

[10] Andrew B. Wolf. 2018. The Impact of Web-Based Video Lectures on Learning in Nursing Education: An Integrative Review. Nursing education perspectives 39, 6 (2018), E16-E20. DOI : http:

//dx.doi.org/10.1097/01.NEP.0000000000000389

[11] GOH Yong-Shian, Sunil Selvarajan, Mui-Lee Chng, Chee-Shiong Tan, and Piyanee Yobas. 2016. Using standardized patients in enhancing undergraduate students' learning experience in mental health nursing. Nurse Education Today 45 (2016), 167-172. 